\title{
Research Hotspots Analysis of China's Knowledge Integration(1999-2013)
}

\author{
Liu Yan-fang \\ School of Management \\ Harbin Normal University \\ Harbin 150025 \\ China
}

\author{
Du Miao \\ School of Management \\ Harbin Normal University \\ Harbin 150025 \\ China
}

\begin{abstract}
The paper divides the development of China' s knowledge integration research into three time periods by the analysis of the data in CAJD database(1999-2013),builds keyword co-occurrence networks of different development stages of knowledge integration research by using Excel and Ucinet,and analyzes research topics of each time period.The article attempts to reveal the knowledge structure and research hotspots in this area,in order to provide a reference for China' $s$ knowledge integration research and the development of practice.
\end{abstract}

Keywords-knowledge integration;co-words analysis;social network analysis;co-authors analysis

Today is the era of knowledge economy,single knowledge can not really help the organization succeed,therefore, the integration of knowledge has been recognized by more and more scholars and experts. This study attempts to have a statistical analysis of the current results in terms of the combination of qualitative and quantitative,and a comprehensive understanding of the development process of China' $s$ knowledge integration research in the past fifteen years and reveal the knowledge structure and research focus of China' s knowledge integration areas.

\section{DATA SOURCE AND RESEARCH METHODS}

\section{A. Data source}

China Academic Journal Network Publishing Database is the world's largest China Academic Journal Full-text data base which can update continuously and dynamically, therefore,the paper choose this database as the data source.Retrieval date was November 11, 2013, and the theme was "knowledge integration", the author eliminated the irrelevant documents from the total 861 documents,obtained 289 valid documents, then extracted keywords, author,posting journals and other fields for statistical analysis.

\section{B. Co-word Analysis}

In 1986, CNRS's Callon $M$ and Law J published the first academic monographs on co-word analysis[1].The basic principle of co-word analysis is:To make a statistics of the keyword frequency which can express the research topics of the field, establish the word matrix based on this,analyse the word matrix and then determine the relationship of affinity between the various topics in the field,sum up and conclude the main structure and research focus of the discipline[2].The co-word matrix in this article is established by means of Excel.

\section{Social Network Analysis}

"The Social Network" refers to the social actors and a collection of their relationship,a social network is a collection which consists of of connections between a number of points and each point[3].Social Network Analysis can trace back to the earliest research in psychology and anthropology of the 1930s.Its research focuses on the analysis of the overall networks structure and the relationships among actors, the method can effectively integrate quantitative data,qualitative data and chart data[4].In this paper,the social network graphs are mainly generated by means of Ucinet software.

\section{Co-authors analysis}

Co-author analysis allows the researchers to have a better understanding of the authors' situation, so as to provide material for revealing the research hotspots of this area.The essence of co-author analysis is to study the relationship among the co-authors, The social network analysis happens to be a network model which focuses on the relationship among actors, node represents acoauthor,connection represents the relationship among the actors.

\section{STATISTICS AND ANALYSIS}

\section{A. Time distribution of literatures}

The annual distribution of the above-mentioned 289 valid documents is shown in Fig.2.As can be seen,our journal articles about knowledge integration research started in 1999, from 1999 to 2001, the research on this topic was very few,but since 2001, the paper amount of 
knowledge integration increased year by year, peaked in 2006 and declined in 2007, then entered the rising period in the next two years,reached a new peak in 2009, and the total amount of the literatures remained stable from 2009 to 2013.The article should be pointed out that the data collection ending on November 11,2013, in fact,the amount of papers in 2013 may have slight variations.

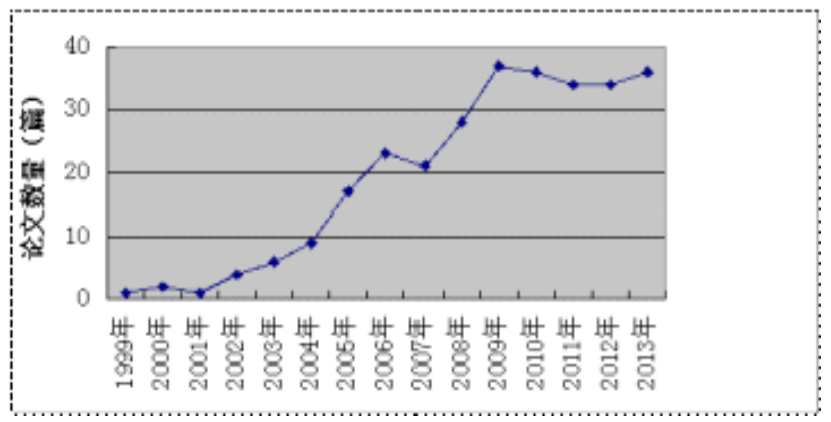

Figure 1. Time distribution graph of knowledge integration literature

\section{B. The statistics and analysis of high frequency words at each time}

The author thinks, the development of China' s knowledge integration can be divided into three time periods:1999-2003(initial period),2004-2008(development period),2009-2013(stable period).

1) Analysis of China's knowledge integration research hotspots at initial period

During this period,the number of the papers about knowledge integration was small and the keywords frequency was very low,therefore,the author didn' t draw the knowledge map.The paper tries to illustrate the research findings in this period with the keywords and the main content of the articles.

Studies of this phase can be summarized as:(1)Definition and characteristics of knowledge integration ability.Hu Chudong(1999)pointed out that we can grasp the basic characteristics of knowledge integration from the unity of macro and micro,technical and non-technical and other different aspects[5].(2)Knowledge integration and knowledge management.Ren Hao and Deng Sanhong (2002)classified knowledge integration manners into form integration,classification integration,three-dimensional integration and purpose integration, then emphasizes that knowledge integration plays a key role in the effect of knowledge management[6].(3)Knowledge integration and knowledge alliance.Cheng Wenyan (2003) considered that the library can integrate knowledge within the organization by integrating existing information systems, human resources and other ways, can implement knowledge alliance program outside the organization by actively developing self knowledge base of libraries, select the appropriate partners and other measures[7].

This shows that the research literature of initial stage focused primarily on the level of connotations, characteristics,significance of knowledge integration and related concepts, and the researches about other aspects were few.
2) Analysis of China's knowledge integration research hotspots at development period

The paper tries to refer to the words and low-frequency words which was proposed by Donohue in 1973[8], combine the actual situation of the data,finally,select 37 keywords whose word frequency was equal or greater than 2 , construct keyword co-occurrence matrix(see Table 1)by Excel software,then import the matrix into social network analysis software Ucinet6.2, and map out keywords network diagram of knowledge integration literature at development stage (shown in Fig.3).

TABLE I. LITERATURE KEYWORDS CO-OCCURRENCE MATRIX OF 2004-2008(PARTIAL)

\begin{tabular}{|c|c|c|c|c|c|c|c|c|c|c|c|c|c|c|c|}
\hline & 吾憐 & & 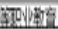 & $5=$ & 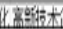 & $A \|$ & Salu & Alest & $8 \operatorname{sins}^{2}$ & 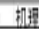 & & & it & & \\
\hline 政都 & & 0 & 0 & & ? & 0 & 0 & 0 & & 0 & 0 & 0 & & 0 & 2 \\
\hline  & & 0 & 0 & & 0 & 0 & 0 & 0 & & 0 & 0 & 0 & & 0 & 0 \\
\hline 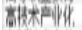 & & 0 & 0 & & 0 & 0 & 0 & 0 & & 0 & 0 & 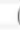 & & 0 & 0 \\
\hline 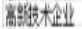 & & 0 & 0 & & 0 & 0 & 0 & 0 & & 0 & 0 & & & 0 & 0 \\
\hline H液 & & 0 & 0 & & 0 & 0 & 0 & 0 & & 0 & 0 & & & l & 0 \\
\hline  & & 0 & 0 & & 0 & 0 & 0 & 0 & & 0 & 1 & & & 0 & 0 \\
\hline B(n) & & 0 & 0 & & 0 & 0 & 0 & 0 & & 0 & 0 & ( & & 0 & 0 \\
\hline Nol & & 0 & 0 & & 0 & 0 & 0 & 1 & & 0 & 0 & ( & & 0 & 0 \\
\hline W & & 0 & 0 & & 0 & 1 & 0 & 0 & & 0 & 0 & ( & & 0 & 0 \\
\hline 解 & & 0 & 0 & & 0 & 0 & 0 & 0 & & 0 & 0 & ( & & 0 & 0 \\
\hline that & & 2 & 0 & & 0 & 0 & 0 & 0 & & 0 & 0 & & & 0 & 0 \\
\hline
\end{tabular}

In Fig.3, each node represents a keyword,different colors represent different $\mathrm{K}$-core,the figures represent cooccurrence frequency of the keywords.K-cores analysis function divide the keywords into four categories, red square and black square nodes occupy the major position of the figure, which represents the hot point of knowledge integration field,and the black square nodes are in the center positon, therefore,the corresponding research focuses are central, and these hotspots can be summarized as follows:(1)Knowledge integration research among different organizations and different groups within the organization.Not difficult to find,the keyword "knowledge integration" is in the most central location in Fig.3, and other studies mainly pivot around “ knowledge integration". .Li Bozhou and Wang Jiankang(2007)pointed out that knowledge integration of multinational conglomerates should note:To identify knowledge property;To build and develop trustful work environment; To dedicate to develop knowledge integration mechanism[9].(2)Knowledge integration research in technology innovation(Keywords for knowledge integration, technological innovation,knowledge management,integrated mode).Shown in Fig.3,the four keywords constitute a $\mathrm{K}$ nuclear cohesive subgroup, and the co-occurrence frequency of keywords " knowledge integration" and "technological innovation" is 9 , which belong to the most in-depth focus of knowledge integration research in this period, and the co-occurrence frequency of knowledge integration and knowledge management is 7 , which are sub-core research focuses.Xie Hongming(2008) considered that the ability to cooperate of knowledge integration ability need to treat the knowledge integration effect as an intermediary, in order to produce a positive impact on technological innovation[10].(3)Research on knowledge sharing and knowledge integration(Keywords for knowledge sharing,knowledge transfer,knowledge 
integration,cooperation innovation).Figure 3 shows, keywords knowledge sharing, knowledge transfer, knowledge integration and cooperation innovation constitute another K-nuclear condensation subgroup,and the relationship of knowledge sharing and knowledge integration is closer,which indicate that knowledge sharing and knowledge integration is one of the study hotspots in this period.Ke Jianglin(2007)recommends that companies should pay attention to knowledge sharing and integration of members and specific strategies in managing $\mathrm{R} \& \mathrm{D}$ team to strengthen the cultivation o team' s social capital[11].

Compared with the initial phase,China' s knowledge integration research has made great progress at development stage.First,the research height has increased gradually from the conceptual elaboration of knowledge integration to specific measures research of knowledge integration among the different organizations and different groups within the organization.Secondly, the researchers' horizons are more open, research perspective is more unique ,and they began to research knowledge integration,organizational performance and technology innovation together.Again,in addition to the above research focuses,knowledge transfer(including keywords knowledge transfer,explicit knowledge,tacit knowledge)research has gradually become the focus of experts and scholars.



Figure 2. Literature keywords social network map of 2004-2008

\section{3) Analysis of China's knowledge integration research hotspots at stable period}

Identical to the above principle,the keywords cooccurrence matrix of this stage is shown in Table 2 , and the knowledge network map is shown in Figure 4.

TABLE II. LITERATURE KEYWORDS CO-OCCURRENCE MATRIX OF 2009-2013(PARTIAL)

\begin{tabular}{|c|c|c|c|c|c|c|c|c|c|c|c|c|c|}
\hline & Cors & A &  & & $I$ & If & & & 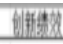 & & & & \\
\hline COBS & 0 & 0 & 0 & 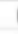 & & 0 & 0 & & 0 & 1 & 0 & 0 & 0 \\
\hline Hin & 0 & 1 & 0 & & & 1 & 1 & & 1 & 0 & 0 & 0 & 0 \\
\hline 产常科合作 & 0 & 0 & 0 & & & 0 & 0 & & 0 & 0 & 0 & ! & 0 \\
\hline$\ddot{\mu} \mathbb{k l}$ & 0 & 0 & 0 & & & 0 & ? & & 0 & 0 & 0 & 0 & 0 \\
\hline 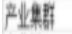 & 0 & 1 & 0 & & & 0 & 0 & & ? & 1 & 0 & 0 & 0 \\
\hline 产晋 & 0 & 0 & 0 & & & 0 & 0 & & 0 & 0 & 0 & 0 & 0 \\
\hline 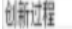 & 0 & 1 & 0 & & & 2 & 0 & & 0 & 0 & 0 & 0 & 0 \\
\hline 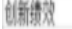 & 1 & 0 & 0 & & & 1 & 0 & & 0 & o & 0 & 0 & 0 \\
\hline 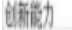 & 0 & 0 & 0 & & & 0 & 0 & & 0 & 0 & ? & 0 & 0 \\
\hline t静佣 & 0 & 0 & 1 & & & 0 & 0 & & 0 & 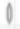 & 0 & 0 & 0 \\
\hline 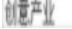 & 0 & 0 & 0 & & 0 & 0 & 0 & & 0 & 0 & 0 & 0 & 0 \\
\hline
\end{tabular}

By the observation and analysis of Figure 4, the , the main research directions of China's knowledge integration from 2009 to 2013 can be divided as
follows:(1)The relationship research of knowledge integration, innovation performance and absorptive capacity (keywords include innovation performance, absorptive capacity, knowledge integration, knowledge integration capability, knowledge integration mechanisms).As in Figure 4, the five words constitute a condensation subgroup of three nuclear , and innovation performance is in the central position.The empirical results of Jian Zhaoquan and Zhan Sunfu(2009) showed that:The absorption capacity had a significant positive effect on knowledge integration;Knowledge integration had a significant positive impact on technology transfer performance;Absorptive capacity had a significant positive impact on technology transfer performance[12].Wu Junjie and Dai Yong (2013)suggested that the ability to integrate knowledge played a corresponding intermediary role in enterprise technology innovation performance[13].(2)The relationship research of ability to integrate knowledge, core competencies and knowledge integration(including keywords knowledge integration capability,knowledge integration, core competencies, organizational learning,and corporate culture).Chen Jianxun(2009)pointed out that knowledge integration played a positive regulative role in the relationship between external social capital and core competencies[14].(3)Influencing factors of knowledge integration(including keywords knowledge integration,structural equation modeling,research universities, and knowledge innovation).As can be seen from Figure 4,the keyword knowledge integration is in the core position,and knowledge innovation,structural equation modeling and other keywords are closely connected to knowledge integration.During this period, the researchers would has a quantitative analysis of data acquired mainly by combining structural equation modeling statistical software to explore the influence factors of knowledge integration.The findings of Liu Yanfang and Yuan Yongjiu(2013)showed that:Different knowledge characteristics had different effects on knowledge integration,not only included positive promotion,but also negative obstacles; Integration willingness of knowledge body,integration ability, and the social networks relationship all had a positive role in promoting integration[15].

Compared with the previous stage, Research at stable period had a great progress.In the first place,the research angles became more diverse and the content became deeper.For example,the supplier knowledge integration research based on radical product innovation.The next,research methods had been more diverse.The experts and scholars began to research by using social network analysis and structural equation modeling.Lastly,In addition to knowledge management,knowledge sharing and other hot issues which still were concerned by researchers,knowledge integration research in collaborative innovation was becoming a new theme discussed by researchers. 


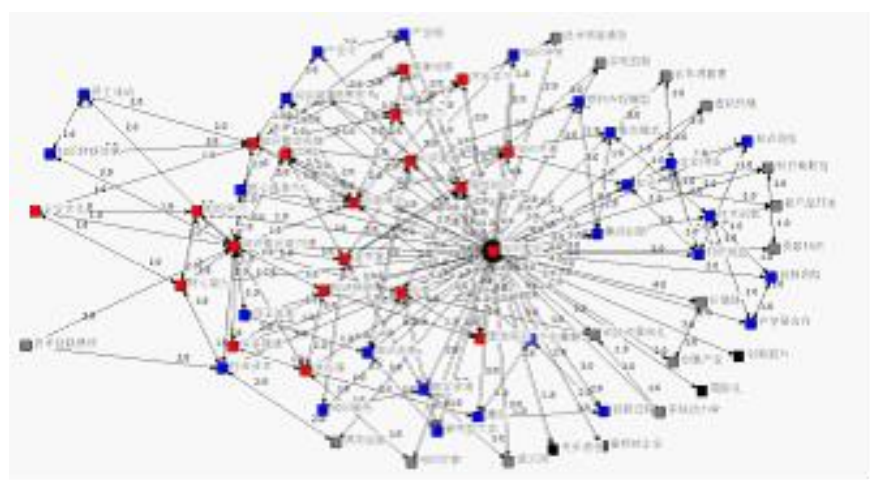

Figure 3. Literature keywords social network map of 2009-2013

Through the analysis of the above academic cooperation groups, we found that,in recent years, the scholars in knowledge integration field focused primarily on knowledge integration model, integration mechanisms, integrating mechanism,influential factors, and other aspects, but also particularly keen on knowledge integration, the relationship among innovation performance and core competencies,knowledge integration methods and enterprise innovation ability.

\section{THE CONCLUSION}

Knowledge integration is a source of competitive advantage for the organization.Organizations need to effectively integrate the available knowledge,maximize its value, in order to make itself has a long-term foothold in the more competitive environment.This paper,through Excel and Ucinet software,tries to reveal the knowledge structure and research focus of China's knowledge integration from different angles, in order to provide a reference for the follow-up study of knowledge integration.

\section{ACKNOWLEDGEMENT}

This work was financially supported by the social science foundation of Heilongjiang

province(13D040),Postdoctoral science foundation of Heilongjiang province(LBH- Z13068) and the Academic backbone foundation of Harbin Normal University (KGB201206).

\section{REFERENCES}

1] Qin Changjiang \& Hou Hanqing.Knowledge Mapping-New areas of information management and knowledge management[J].Journal of Academic Libraries, 2009, (1): 30-37.

[2] Zhang Qin \& Ma Feicheng.Foreign knowledge management paradigm-Based on co-word analysis method[J].Journal of Management Sciences in China,2007,06:65-75.

[3] Liu Jun.Lectures on Whole Network Approach.[M].Shanghai:Gezhi Publishing House, 2009:01-02.

[4] Li Yaoqi \& Xie Lishan.The application and Prospect of Social Network Analysis in organization management research[J].Chinese Journal of Management,2013,01:146-154.

[5] $\mathrm{Hu}$ Chudong.Discussion about knowledge integration ability[J].Jianghan Tribune,1999,05:34-36.

[6] Ren Hao \& Deng Sanhong.Important step in knowledge management-knowledge Integration[J]. Information Science, 2002,06:650-653.

[7] Cheng Wenyan.Talking library knowledge management solutionsknowledge integration and knowledge alliance[J].Information Science,2003,04:395-397

[8] Donohue J C.Understanding Scientific Literature:A bibliographic approach[M].Cambridge:The MIT Press, 1973:49.

[9] Li Bozhou \& Wang Jiankang.Research on knowledge integration mechanism of multinational group[J].Science \& Technology Progress and Policy,2007,04:156-159.

[10] Xie Hongming,Wu Su \& Wang Xianbiao.Knowledge integration ability,effect and technological innovation[J].Science of Science and Management of S.\& T.,2008,08:88-93.

[11] Ke Jianglin,Sun Jianmin,Shi Jintao \& Gu Qinxuan.An empirical research on the relationship between social capital and team performance of enterprise R \& D team--with knowledge sharing and knowledge integration as mediator[J].Management World,2007,03:89-101.

[12] Jian Zhaoquan \& Zhan Sunfu.Relationship research on the relationship among absorptive capacity,knowledge integration,organization knowledge, and technology transfer performance $[\mathrm{J}]$. Science of Science and Management of S.\& T.,2009,06:81-86.

[13] Wu Junjie \& Dai Yong.Relationship research on entrepreneurial social capital, knowledge integration capability and technology innovation performance[J].Science \& Technology Progress and Policy,2013,11:84-88.

[14] Chen Jianxun,Pan Changcai \& Wu Longzeng.The impact of external social capital on the core competence--the regulatory role of knowledge integration[J].Studies in Science of Science, 2009,02:244-249.

[15] Liu Yanfang \& Yuan Yongjiu.Empirical Study on effect factors of knowledge integration in research universities oriented to Knowledge Innovation.[J].Science \& Technology Progress and Policy,2013,07:139-145. 